\title{
Note on translation, transliteration and dates
}

For the transliteration of Russian terms and proper names we have used the simplified library of Congress system without diacritics, apart from (") to indicate the soft sign (ь). Terms and names in Central Asian languages are generally transliterated from historic spellings in the Arabic script. With the exception of Daniel Prior's transcription of Musa Chaghatay uulu's poetry, where absolute accuracy was essential, we have generally favoured comprehensibility for non-specialists and ease of reading and minimised the use of diacritics and non-standard characters. Where familiar versions of a name already exist in English we have stuck with the established spelling (e.g., Samarkand not Samarqand). For the same reason we use "Kazakh" and "Kyrgyz" rather than the more correct "Qazaq" and "Qirghiz", except when referring to the language rather than the people. Where a direct English equivalent exists, technical terms have been translated, notably oblast' (province) and uezd (district). All translations are the authors' own unless otherwise indicated.

Before 14 February 1918, the Russian Empire operated according to the Julian calendar, which was thirteen days behind the Gregorian calendar used in the rest of Europe, and now worldwide. All dates in the text and references of this volume are old style (OS) up to the point when the calendar changed, reflecting those in the sources. 